\title{
Follow up of focal narrowing of retinal arterioles in glaucoma
}

Konstantinos I Papastathopoulos, Jost B Jonas

\begin{abstract}
Aim-To evaluate whether focal narrowing of retinal arterioles increases with progressive glaucomatous optic neuropathy.

Methods-Focal narrowing of retinal arterioles and area of neuroretinal rim were morphometrically evaluated on colour stereo optic disc photographs of 59 patients with primary open angle glaucoma, 22 patients with normal pressure glaucoma, 11 patients with secondary open angle glaucoma, and 31 patients with ocular hypertension. Minimum follow up was 8 months. Focal arteriolar narrowing was quantified by calculating the ratio of the vessel width in the broadest to the narrowest vessel part.
\end{abstract}

Results-In the subgroup of patients with progressive glaucomatous optic nerve damage $(n=37)$, focal narrowing of retinal arterioles increased significantly $(p<0.005)$ with decreasing neuroretinal rim area. In the subgroup of patients with stable appearance of the optic disc $(n=86)$, focal narrowing of retinal arterioles did not change significantly $(p=0.79)$. The positive correlation between increasing focal thinning of retinal arterioles and progression of glaucomatous optic neuropathy was present, although not statistically significant, in all the glaucoma subtypes examined. The location of focal thinning of retinal arterioles did not change in the follow up.

Conclusions-Focal narrowing of retinal arterioles increases significantly with progressive glaucomatous optic neuropathy, independent of the type of glaucoma. It is stable in patients with non-progressive glaucoma. The findings agree with previous reports on a higher degree of focal arteriole narrowing in eyes with pronounced optic nerve damage in comparison with those with moderate optic nerve atrophy or normal eyes. In the clinical management of patients with glaucoma, in some eyes, increasing focal arteriole narrowing may suggest progression of disease.

(Br f Ophthalmol 1999;83:285-289)

Accepted for publication 30 September 1998

Table 1 Demography of the study groups (mean (SD))

\begin{tabular}{lllll}
\hline & $\begin{array}{l}\text { Patients with } \\
\text { ocular } \\
\text { hypertension }\end{array}$ & $\begin{array}{l}\text { Patients with } \\
\text { primary open } \\
\text { angle glaucoma }\end{array}$ & $\begin{array}{l}\text { Patients with } \\
\text { normal pressure } \\
\text { glaucoma }\end{array}$ & $\begin{array}{l}\text { Patients with } \\
\text { secondary open } \\
\text { angle glaucoma }\end{array}$ \\
\hline Patients (n) & 31 & 59 & 22 & 11 \\
Age (years) & $44.5(10.8)$ & $46.6(11.7)$ & $53.9(9.7)$ & $41.4(14.6)$ \\
Women/men & $18 / 13$ & $23 / 36$ & $13 / 9$ & $1 / 10$ \\
Refractive error (D) & $-0.3(1.6)$ & $-0.7(2.2)$ & $0.25(2.8)$ & $-2.1(3.2)$ \\
\hline
\end{tabular}

Eyes with optic nerve damage, independent of the pathogenesis of optic nerve atrophy, show a diffuse decrease in the diameter of the retinal arterioles $^{1-3}$ corresponding to haemodynamic changes in the retina and optic disc. ${ }^{4-11}$ Rader et $a l,{ }^{12}$ Rankin and Drance, ${ }^{13}$ and Papastathopoulos and Jonas ${ }^{14}$ have described the additional occurrence of focal narrowing of retinal arterioles in eyes with glaucomatous or nonglaucomatous optic nerve atrophy. Frequency and degree of such focal narrowing correlated positively with the degree of optic nerve damage and age. Patients with advanced optic neuropathy had a more pronounced focal arteriole narrowing than patients with moderate optic nerve damage. In a recent fluorescein angiographic study, ${ }^{15}$ focal narrowing of the retinal arterioles, as detected by ophthalmoscopy, represented a stenosis of the vessel lumen on fluorescein angiograms.

The present prospective follow up investigation was performed to determine whether focal narrowing of retinal arterioles in glaucoma is stable or whether it varies with progression of the disease, and whether a correlation between focal arteriole narrowing and progression of glaucomatous optic neuropathy is found in various types of open angle glaucoma.

\section{Material and methods}

The study included 59 patients with primary open angle glaucoma, 11 patients with secondary open angle glaucoma, 22 patients with normal pressure glaucoma, and 31 subjects with ocular hypertension (Table 1). These were all the glaucoma patients whose follow up optic disc photographs were readily available in the laboratory at the time the study was performed. There was no other selection criterion. The patients were examined as part of an ongoing prospective and longitudinal study on the biomorphometry of the optic nerve. The various study groups did not differ significantly in age (Table 1). Eyes with a myopic refractive error exceeding -8 dioptres were excluded owing to a differing optic disc morphology. ${ }^{16}$ All patients had at least one follow up examination, including day and night intraocular pressure profile, and colour stereo optic disc photographs. The interval between the first examination and last examination was at least 8 months (median 37 months; range 8-93 months).

Criteria for the diagnosis of open angle glaucoma, each of which had to be fulfilled, were an open anterior chamber angle; and glaucomatous changes of the optic nerve head, such as unusually small neuroretinal rim area in relation to optic disc size, abnormal shape of 
the neuroretinal rim, and cup/disc ratios being vertically greater than horizontally; and localised or diffuse defects of the retinal nerve fibre layer. The whole glaucoma group was divided into eyes affected by primary open angle glaucoma in which no obvious reason for an elevation of intraocular pressure could be detected; eyes with secondary open angle glaucoma; and eyes with normal pressure glaucoma with intraocular pressure readings of $21 \mathrm{~mm} \mathrm{Hg}$ or less (Table 1). Elevation of intraocular pressure in the patients with secondary open angle glaucoma was due to pigmentary glaucoma $(n=7)$, topical use of cortisone $(n=2)$, pseudoexfoliative glaucoma $(n=1)$, and traumatic recession of the anterior chamber angle $(n=1)$. The patients with pigmentary glaucoma showed translucent radial defects in the peripheral iris pigment epithelium, Krukenberg's spindle, and hyperpigmentation of both the hyaloid capsular ligament and anterior chamber angle. Pseudoexfoliation of the lens was characterised by a dandruff-like material on the lens zonules and lens surface especially in its centre and the periphery separated by an intermediate clear zone, a secondary pigment dispersion with translucent defects in the parapupillary region of the iris pigment epithelium, often a hyperpigmentation of the anterior chamber angle, and relatively poor pupil dilatation. For the diagnosis of normal pressure glaucoma, the intraocular pressure had to be no more than 21 $\mathrm{mm} \mathrm{Hg}$ for at least two day and night pressure profiles. Neurological, neuroradiological, and general medical examination had to reveal no reason for optic nerve damage other than glaucoma. Patients with intraocular pressure readings of more than $21 \mathrm{~mm} \mathrm{Hg}$, an unremarkable appearance of the optic disc, and a normal visual field were regarded as ocular hypertensives.

For all eyes, 15 degree colour stereo optic disc transparencies had been taken using a telecentric fundus camera. The disc slides were projected in a scale of 1 to 15 . The outlines of the optic cup and optic disc were plotted on paper and morphometrically analysed. To obtain values in absolute size units-that is, $\mathrm{mm}$ or $\mathrm{mm}^{2}$, the ocular and photographic magnification was corrected using Littmann's method. ${ }^{17}$ The method has already been described in detail. ${ }^{2} 3$ To compensate for the individual variation in optic disc size, we calculated two additional neuroretinal rim variables - the rim area/disc area ratio, and the neuroretinal rim area defect. As already described, ${ }^{18}$ the neuroretinal rim area defect was calculated as:

neuroretinal $\mathrm{rim}$ area defect $=$ expected $\mathrm{rim}$ area - measured rim area

"Expected rim area" was defined as: expected rim area $=0.131 \times$ optic disc area + 1.384 .

The latter was the previously determined equation of the linear regression for the correlation between neuroretinal rim area and optic disc area in normal subjects. ${ }^{18}$

The photographs of the first and last examinations were simultaneously projected. The assessment of progression of the glaucomatous optic nerve atrophy was performed independently by two observers by comparing the two optic disc photographs in a masked manner, without knowledge of the clinical diagnosis and the visual field data, and without knowing which one of the two stereo pairs of photographs had been taken first. Criteria for progression of the glaucomatous optic neuropathy were focal or diffuse diminution of the neuroretinal rim, which was present in all progressive eyes included in the study, and, additionally, enlargement of parapapillary chorioretinal atrophy, the new occurrence of retinal nerve fibre layer defects, enlargement of pre-existing retinal nerve fibre layer defects, or the presence of an optic disc haemorrhage in the last optic disc photograph.

Focal narrowing of the retinal arterioles was examined independently in a second step, again by projecting simultaneously the first and the last optic disc transparencies in a masked fashion. After initial defocusing of the projector, the intrapapillary region was covered and the projector was refocused again. The narrowest and the broadest parts of the vessel, at a distance of about $0.1-0.5 \mathrm{~mm}$ to each other, were directly measured with a millimetre scale at the same retinal position on both photographs. These narrow vessel sections were circumscribed with an abrupt transition to the broader part of the vessel (Figs 1 and 2). Owing to an irregular course of the vessels, the area of the peripapillary scleral ring and the intrapapillary region were not taken into account. Focal vessel narrowing was evaluated in the region extending from the disc border to the margin of the photograph and quantified by calculating the ratio of the broadest to the narrowest vessel part. Only one randomly selected eye per patient was used for statistical analysis.

The present study was started after more than 800 photographs not included in the present study had been evaluated. The present study is the continuation of a previous cross sectional investigation on focal thinning of retinal arterioles. ${ }^{14}$ For the present study with quantitative grading of focal vessel narrowing, we determined the coefficient of variation by remeasuring 20 photographs 10 times each. For each re-evaluation, the photographs were remixed. The coefficient of variation calculated as ratio of the mean of the standard deviations divided by the mean of the means was 0.058 .

\section{Results}

The group of patients with progression of glaucomatous optic nerve atrophy consisted of 37 patients. The patients with progression of glaucomatous optic neuropathy and the patients without detected progression did not vary significantly in age $(\mathrm{p}=0.20)$ or the type of antiglaucomatous medication - that is, the proportion of patients taking topical $\beta$ blockers, dorzolamide, miotics, latanoprost, and acetazolamide did not vary significantly ( $p>0.20 ; \chi^{2}$ test, Fisher's exact test) between the progressive group and the non-progressive group. With patients with diabetes mellitus being excluded from the study, the proportion of 

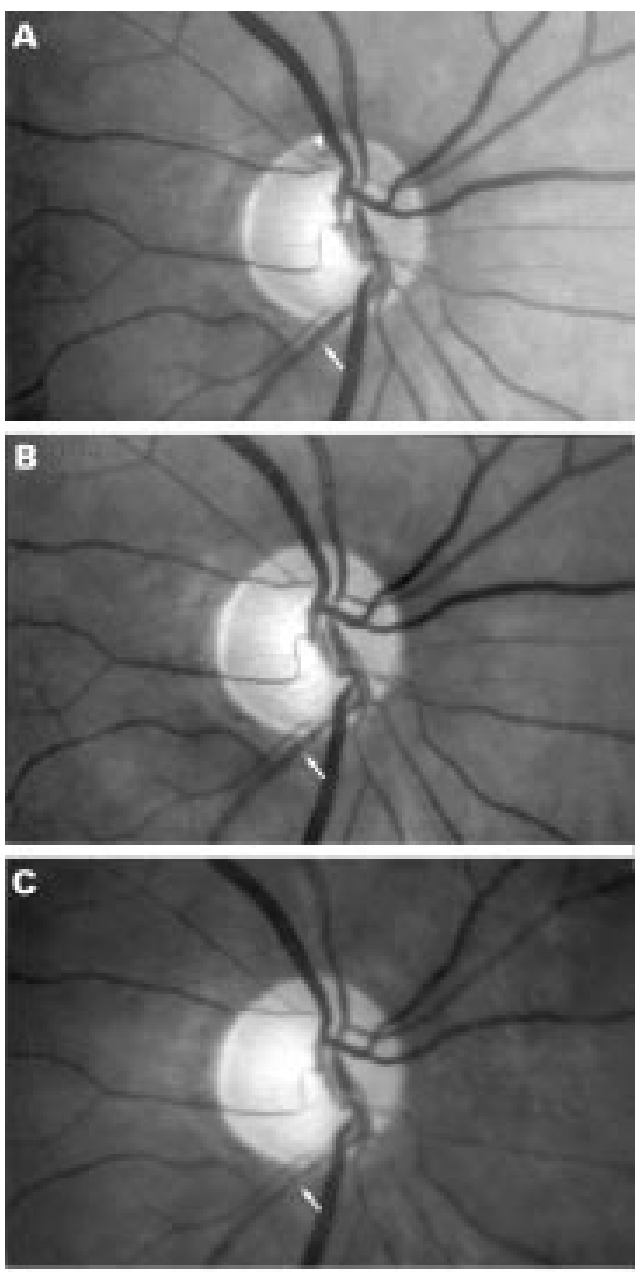

Figure 1 Optic disc photograph with progression of glaucomatous optic neuropathy. First examination (A), follow up examination after 22 months (B), and after additional 30 months $(C)$. Note increase in focal thinning of retinal arteriole (white arrows); white arrowhead: disc haemorrhage.

patients with arterial hypertension, coronary heart disease, and migraine did not vary significantly between the subgroup with progression of glaucomatous optic neuropathy and the subgroup with no detected progression ( $p>0.20 ; \chi^{2}$ test, Fisher's exact test); and it did not vary significantly $(p>0.20)$ between the patients with progressive focal arteriole thinning and the patients with unchanged degree of focal arteriole narrowing. According to the clinical charts and the general medication taken by the patient, and the medical history of the patient, we determined whether the patients were suffering from arterial hypertension, coronary heart disease, or migraine.

Comparing the first examination and the last examination with each other in the subgroup of patients with worsening of the optic nerve, the ratio of the broadest to thinnest vessel part was significantly higher at the last examination than at the first examination (1.34 (SD 0.24) versus 1.26 (0.25); $\mathrm{p}=0.04$; Wilcoxon test). The result corresponded to a higher degree of focal vessel thinning at the last examination. An increasing ratio of broadest to narrowest vessel part correlated significantly $(\mathrm{p}<0.005)$ with decreasing neuroretinal rim area (Fig 3); the
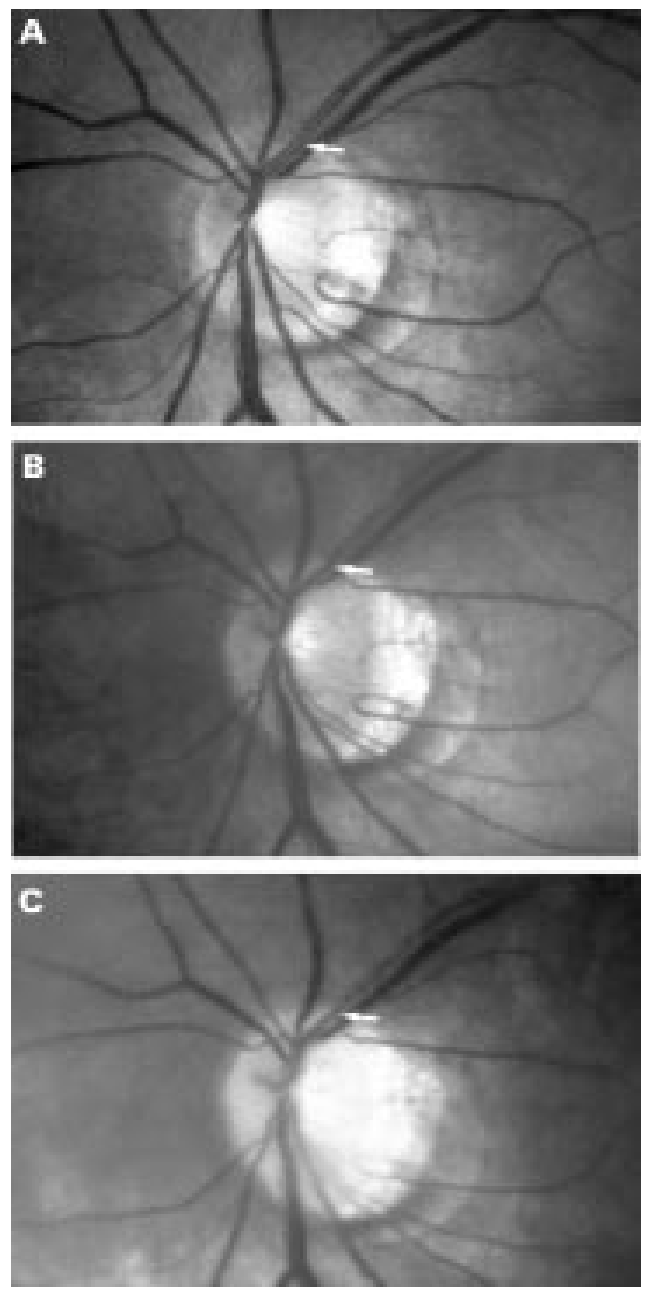

Figure 2 Optic disc photograph with progression of glaucomatous optic neuropathy. First examination (A), follow up examination after 55 months $(B)$, and after a further 47 months $(C)$. Note increase in focal thinning of retinal arteriole (white arrows).

more pronounced the loss of neuroretinal rim, the higher was the increase in focal thinning of the retinal arterioles.

Correlation of the rim area/disc area ratio and the neuroretinal rim area defect with the degree of focal arteriole thinning, gave similar results to those obtained when the neuroretinal rim area was taken for the correlation. Focal arteriole thinning increased significantly with decreasing rim area/disc area ratio $(p<0.007)$ and with increasing neuroretinal rim area defect $(p<0.009)$. These results suggested that optic disc size did not have a major influence on the results of the study.

The increase in arteriole thinning measured as the enlarging ratio of broadest to narrowest vessel part correlated significantly and positively with the time interval between the first and the last examination (correlation coefficient $R=0.40 ; \mathrm{p}<0.05$ ), the longer the follow up period, the more pronounced was the focal narrowing of retinal arterioles. Correspondingly, neuroretinal rim area decreased significantly with increasing duration of the study $(R$ $=0.42 ; \mathrm{p}=0.01)$. If the group with progression was divided in two subgroups depending on the length of follow up, increase in the degree 


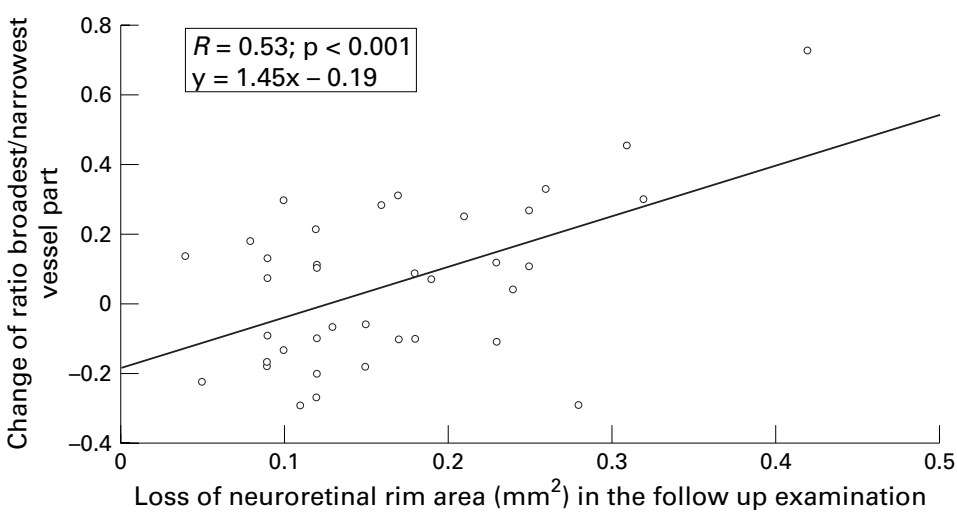

Figure 3 Scattergram showing the correlation between decrease in neuroretinal rim area and increase in focal thinning of retinal arterioles measured as ratio of broadest to narrowest vessel part.

of focal arteriole thinning was significantly $(p<0.01)$ more pronounced in the subgroup with the longer follow up time compared with the subgroup with the shorter follow up period.

In the subgroup of patients with a stable appearance of the optic disc $(n=86)$, the ratio of broadest to narrowest part of the retinal arterioles did not vary significantly between the first and last examination (1.28 (0.19) versus 1.30 (0.22); $\mathrm{p}=0.79$; Wilcoxon test).

In all eyes included in the study the location of focal arteriole thinning did not change in the follow up examination. The positive correlation between increase of focal thinning of retinal arterioles and progression of glaucomatous optic neuropathy was found in all types of open angle glaucoma included in the study. Perhaps, owing to the relative smallness of each of the glaucoma subgroups, the correlation did not become statistically significant in the glaucoma subgroups taken separately.

\section{Discussion}

Generalised narrowing of retinal vessels has been described for both glaucomatous and non-glaucomatous optic neuropathies. ${ }^{1-3}$ In glaucoma, the vessel diameter reduces with decreasing area of the neuroretinal rim, diminishing visibility of the retinal nerve fibre layer, and increasing visual field defects. ${ }^{2}$ Since reduction of vessel calibre is also found in eyes with non-glaucomatous optic nerve damage such as non-arteritic anterior ischaemic optic neuropathy ${ }^{19}$ and descending optic nerve atrophy, ${ }^{13}$ it has been assumed that a generalised reduction of the vessel diameter is typical for optic nerve damage but not characteristic for glaucoma. From a pathogenetic point of view, it suggested that the vessel reduction was not causative for the glaucomatous optic nerve fibre loss but secondary to a reduced demand in the superficial layers of the retina.

Rader and colleagues have drawn attention to focal narrowing of the retinal arterioles in the peripapillary region of eyes with glaucoma or non-arteritic anterior ischaemic optic neuropathy. ${ }^{12}$ Similar observations were made by Rankin and Drance, ${ }^{13}$ and in our department. ${ }^{14}$ These studies showed that, in addition to a diffuse decrease in the diameter of the retinal arterioles, focal narrowing of retinal arterioles was typical for eyes with optic nerve damage. However, focal vessel narrowing was not pathognomonic for glaucoma as a whole group nor specific for special types of the glaucomas such as normal pressure glaucoma. In a recent study, a significant correlation was found between focal narrowing of retinal arterioles on fundus photographs and narrowing of the same retinal arterioles on fluorescein angiograms. ${ }^{15}$ The results suggested that focal vessel narrowing represented a real stenosis of the retinal arterioles, and that it was not purely an ophthalmoscopic artefact. Since flow through a vessel is proportional to the square of the vessel radius, a reduction of the arteriole diameter by $50 \%$ or more, which has been described for eyes with optic neuropathies, ${ }^{12-15}$ may lead to a marked change in blood flow. The findings suggest that focal narrowing of the retinal arterioles may be associated with some of the haemodynamic changes that have already been found in the optic nerve and retina of eyes with glaucoma..$^{4-11}$

In agreement with earlier cross sectional investigations, the present longitudinal study indicates that focal narrowing of retinal arterioles increases in eyes with progressive glaucomatous optic neuropathy. The progressive focal narrowing of the retinal arterioles correlates with the amount of continuing neuroretinal rim loss. Correspondingly, in eyes with a stable appearance of the optic disc, focal narrowing of retinal arterioles did not show a significant change. In the measurement of focal arteriole thinning, one must take into account, that progression of optic neuropathy is also associated with a diffuse decrease of the diameter of retinal arterioles. ${ }^{1-3}$ Owing to the definition of focal arteriole thinning as a ratio of broadest to thinnest vessel part, a diffuse thinning of the retinal arteriole will influence the measurements of focal arteriole narrowing. The observation that the location of focal arteriole thinning did not change in the follow up examination suggests that the vessel narrowing is not the result of a temporary effect induced by the ocular pulse or another short acting mechanism such as vasospasm.

Focal arteriole thinning may be added to the panoply of morphological features which can be found in eyes with glaucoma. Identification of focal arteriole may be useful for the diagnosis of glaucoma and the observation of increasing arteriole thinning may suggest progression of the disease. This, however, may not be valid for the individual patient. One has to take into account, however, that increasing focal arteriolar narrowing does not necessarily suggest progression of glaucoma. Since patients with various types of open angle glaucoma examined in the present study showed a similar relation between increasing focal arteriole thinning and progressive glaucomatous optic neuropathy, focal arteriole narrowing may not be pathognomonic for any particular type of glaucoma. A similar finding has already been reported for diffuse thinning of retinal arterioles. ${ }^{1-3} 19$ Correspondingly, focal arteriole thinning alone should not lead to the diagnosis 
of glaucoma, since focal narrowing of retinal arterioles has been described for several non-glaucomatous optic neuropathies such as non-arteritic anterior ischaemic optic neuropathy. ${ }^{12} 14$

Future studies may show whether, in conjunction with an increase in focal arteriole narrowing, the retinal blood flow decreases in eyes with progressive glaucoma, and whether the relation between increasing focal narrowing of retinal arterioles and progressive optic nerve damage, as shown in the present study on patients with glaucoma, is also valid for patients with non-glaucomatous optic nerve atrophy.

Presented at the Annual Meeting of the American Academy of Ophthalmology, San Francisco, 26-29 October 1997. A2).

Supported by Deutsche Forschungsgemeinschaft (SFB 539/

1 Frisén L, Claesson M. Narrowing of the retinal arterioles in descending optic atrophy. A quantitative clinical study. Ophthalmology 1984;91:1342-6.

2 Jonas JB, Naumann GOH. Parapapillary retinal vessel diameter in normal and glaucoma eyes. II. Correlations. Invest Ophthalmol Vis Sci 1989;30:1604-11.

3 Jonas JB, Fernández MC, Naumann GOH. Parapapillary atrophy and retinal vessel caliber in nonglaucomatous optic nerve damage. Invest Ophthalmol Vis Sci 1991;32:2942-7.

4 Spaeth GL. Fluorescein angiography: its contributions towards understanding the mechanisms of visual loss in towards understanding the mechanisms of visual loss
glaucoma. Trans Am Ophthalmol Soc 1975;73:491-553.

5 Schwartz B, Kern J. Age, increased ocular and blood pressures, and retinal and disc fluorescein angiogram. Arch Ophthalmol 1980;98:1980-6.
6 Nagin P, Schwartz B, Reynolds G. Measurement of fluorescein angiograms of the optic disc and the retina using computerized image analysis. Ophthalmology 1985;92:547-52.

7 Tuulonen A, Nagin P, Schwartz B, et al. Increase of pallor and fluorescein filling defects of the optic disc in the follow up of ocular hypertensives measured by computerized image analysis. Ophthalmology 1987;94:558-63.

8 Robert Y, Steiner D, Hendrickson P. Papillary circulation dynamics in glaucoma. Graefes Arch Clin Exp Ophthalmol 1989;227:436-9.

9 Wolf S, Arend O, Sponsel WE, et al. Retinal hemodynamics using scanning laser ophthalmoscopy and hemorheology in chronic open-angle glaucoma. Ophthalmology 1993;100: 1561-6.

10 Harris A, Sergott RC, Spaeth GL, et al. Color Doppler analysis of ocular vessel blood velocity in normal-tension analysis of ocular vessel blood velocity in no

11 Michelson G, Langhans MJ, Groh MJM. Perfusion of the juxtrapapillary retina and the neuroretinal rim area in primary open-angle glaucoma. F Glaucoma 1996;5:91-8.

12 Rader J, Feuer J, Anderson DR. Peripapillary vasoconstriction in the glaucomas and the anterior ischemic optic neuropathy. Am f Ophthalmol 1994;117:72-80.

13 Rankin SJA, Drance SM. Peripapillary focal retinal arteriolar narrowing in open angle glaucoma. f Glaucoma 1996;5:22-8.

14 Papastathopoulos KI, Jonas JB. Focal narrowing of the retinal arterioles in optic nerve atrophy. Ophthalmology 1995;102:1706-11

15 Papastathopoulos KI, Jonas JB. Fluorescein angiographic correlation of focal narrowing of retinal arterioles in glaucoma. Br f Ophthalmol 1998;82:48-50.

16 Jonas JB, Gusek GC, Naumann GOH. Optic disk morphometry in high myopia. Graefes Arch Clin Exp Ophthalmol 1988;226:587-90.

17 Littmann H. Zur Bestimmung der wahren Größe eines Objektes auf dem Hintergrund des lebenden Auges. Klin Monatsbl Augenheilkd 1982;180:286-9.

18 Jonas JB, Gründler AK. Correlation between mean visual fleld loss and morphometric optic disc variables in the open-angle glaucomas. Am f Ophthalmol 1997;124:488-97.

19 Jonas JB, Xu L. Optic disc morphology in eyes after nonarteritic anterior ischemic optic neuropathy. Invest Ophthalmol Vis Sci 1993;34:2260-5. 